\title{
Mechanical control of tissue-engineered bone
}

\author{
Ben P Hung 1,2, Daphne L Hutton ${ }^{1,2}$ and Warren L Grayson, ,2,*
}

\begin{abstract}
Bone is a load-bearing tissue and physical forces play key roles in the development and maintenance of its structure. Mechanical cues can stimulate the expression of an osteogenic phenotype, enhance matrix and mineral deposition, and influence tissue organization to improve the functional outcome of engineered bone grafts. In recent years, a number of studies have investigated the effects of biophysical forces on the bone formation properties of osteoprogenitor cells. The application of physiologically relevant stimuli to tissue-engineered bone may be determined through observation and understanding of forces to which osteoblasts, osteoclasts, and osteocytes are exposed in native bone. Subsequently, these cues may be parameterized and their effects studied in welldefined in vitro systems. The osteo-inductive effects of three specific mechanical cues - shear stress, substrate rigidity, and nanotopography - on cells cultured in monolayer or in three-dimensional biomaterial scaffolds in vitro are reviewed. Additionally, we address the time-dependent effects of mechanical cues on vascular infiltration and de novo bone formation in acellular scaffolds implanted into load-bearing sites in vivo. Recent studies employing cutting-edge advances in biomaterial fabrication and bioreactor design have provided key insights into the role of mechanical cues on cellular fate and tissue properties of engineered bone grafts. By providing mechanistic understanding, future studies may go beyond empirical approaches to rational design of engineering systems to control tissue development.
\end{abstract}

\section{Introduction}

Bone tissue engineering (BTE) has the potential to make tremendous clinical impact for the repair and treatment

*Correspondence: wgrayson@jhmi.edu

'Department of Biomedical Engineering, The Johns Hopkins University, Baltimore, MD 21287, USA

Full list of author information is available at the end of the article of massive bone loss. While autografts are the current gold standard for treatment, limitations to this approach include tissue availability and donor-site morbidity. Allografts, meanwhile, require the use of immunosuppressive drugs and carry the risk of disease transmission. In contrast, engineered grafts may utilize autologous cell sources with little co-morbidity and can be used to treat critical-sized bone defects. Traditionally, BTE has combined cells with biomaterial scaffolds and osteoinductive biological factors to guide the development of cells into tissue grafts. Initial studies demonstrated cellular expression of bone-specific proteins but the grafts inevitably lacked adequate mechanical properties needed to withstand physiological loads. This shortcoming has been addressed by incorporating biophysical cues into the culture environment. At the most fundamental level, it is critical to understand the mechanism(s) through which cells in native bone are influenced by mechanical cues. Then, guided by the biomimetic principle [1], it may be possible to determine which forces are most effective for developing bone grafts with superior mechanical properties. Even so, knowledge regarding the effect of timing, dose and loading protocols of mechanical stimuli on cells cultured within three-dimensional scaffolds has primarily been determined empirically. Using tissue-culture bioreactors, various biophysical forces have been applied to developing constructs. These forces enhance the expression of an osteogenic phenotype in cells embedded within the scaffold resulting in increased production and organization of the extracellular matrix (ECM) and increased mineral deposition. In this article, we review how our current understanding of the microanatomy of native bone and cellular mechanotransduction has impacted the application of mechanical forces in biomimetic tissue engineering approaches.

\section{Native mechanics of bone}

Bone actively and continuously remodels in response to physiological loading. Studies have found that strains experienced by bone tissues due to everyday activity range from $0.1 \%$ to $0.35 \%$ [2]. Strains above this range (but below the yield point) lead to bone strengthening while sub-physiological strains lead to bone resorption [2-4]. Three major cell types mediate remodeling: osteoblasts (which deposit new bone matrix), osteocytes 
(which are encased in mineral), and osteoclasts (responsible for bone resorption), and it is the coordinated activity of these cells that enable the coupling of bone structure and function. There is evidence that mechanical stimuli influence the proliferation and function of osteoclasts and osteoblasts in a spatiotemporal manner: bone regions experiencing high strains exhibit significant reduction in osteoclast proliferation [5]. Conversely, simulated microgravity conditions have been shown to suppress osteoblast function and numbers [6].

Osteocytes, however, comprise the majority of cells in compact bone, and are the cells primarily responsible for transducing biophysical signals into specific biological responses in bone. The anatomical location of the osteocytes, encased within lacunae, enable them to 'sense' physiological loads. Compressive loading of bone (for example, during walking) results in non-uniform strains macroscopically. The associated volume and pressure differences within the interconnected canalicular network cause interstitial fluid flow, which imparts shear stresses on the order of 1 to $3 \mathrm{~Pa}$ to the osteocytes [2,7-9]. This conversion from strain to shear stress amplifies the stimulus received by cells [7] and osteocytes transduce these signals through stretch-activated ion channels [10] and via the primary cilium [11]. As a result, bone cells respond to dynamic stimuli [12,13]; a static load produces an initial pressure gradient, which the resulting fluid flow returns to equilibrium, halting further flow, and abolishing the stimulus. The interconnectivity of osteocytes through canaliculi provides an excellent relay network for transmitting mechanical and biochemical signals to osteoblasts and osteoclasts that reside on the endosteal surface [8]. Exquisite in vitro studies, however, have demonstrated that two other physical signals may play a role in directing the activity of osteoprogenitors: the stiffness and topography of the substrate on which they reside. As a result, recent studies regarding the role of mechanics in BTE have focused primarily on the effects of shear stress, substrate rigidity and nanotopography in directing cellular differentiation and enhancing the mechanical competence of engineered bone grafts (Figure 1).

\section{Role of perfusion-mediated shear stress (two- and three-dimensional)}

BTE has made extensive use of bone marrow-derived human mesenchymal stem cells (MSCs) and the effects of mechanical cues have primarily been studied using these cells and osteoblastic cell lines. Rigorous studies into the osteogenic effects of shear stress have been performed using monolayer cultures in parallel plate chambers, as these configurations facilitate accurate measurements of actual shear forces experienced by cells. Osteogenic cells exhibit both dose- and time-dependent changes in gene expression in response to shear forces. Comparisons of oscillatory and pulsatile shear profiles in comparison to steady shear stresses revealed that pulsatile shear elicited the greatest osteogenic response [14]. This result is somewhat surprising given that physiological loading and unloading during walking and running result in oscillatory profiles. Other studies [15] have shown that oscillatory shear elicits anti-osteoclastic responses from osteocytes as evidenced by upregulation of cyclooxygenase-2 (COX-2), downregulation of receptor activator of nuclear factor $\mathrm{kB}$ ligand (RANKL), and downregulation of osteoprotegerin (OPG). This effect is enhanced at high stress amplitudes (approximately $5 \mathrm{~Pa}$ ), high frequencies $(2 \mathrm{~Hz})$, and long loading durations (4 hours). Interestingly, while each of these parameters alone enhances osteogenesis, it was unclear how they interact. For instance, stress applied at $5 \mathrm{~Pa}$ and $0.5 \mathrm{~Hz}$ had a greater effect than did stress applied at $5 \mathrm{~Pa}$ and $2 \mathrm{~Hz}$. It is clear that further work is required to understand the effect of oscillatory shear parameters on osteogenesis.

Shear stress also impacts cellular behavior in threedimensional scaffolds. Previous studies have investigated the effects of superficial flow velocities on the osteogenic profile of MSCs grown in porous scaffolds [16-19]. Due to differences in cell types, scaffold types, and bioreactor designs, the results from these various studies cannot be directly compared. It is nevertheless evident that flow velocities can be optimized based on ECM and mineral deposition. An order of magnitude estimation found that shear stress in these systems is likely to be in the $\mathrm{mPa}$ range, two to three orders of magnitude lower than reported values for native bone. More rigorous computational fluid dynamics analyses for a similar system reported similar values [20]. Interestingly, subsequent studies also demonstrated correlations between tissue structure and organization within the graft and flow patterns [21].

In spite of these data, it is considerably more challenging to identify the effects of flow-induced shear stress on tissue formation in three-dimensional scaffolds. Firstly, perfusion enhances nutrient and oxygen transport to cells in three-dimensional cultures via convection, making it difficult to decouple the beneficial effects of shear from those of improved mass transport. Additionally, complex flow patterns through the internal scaffold geometry result in complicated shear distribution profiles, making it challenging to correlate specific shear forces with cellular outcomes. This difficulty was partially overcome in three-dimensional systems by varying the medium viscosity while keeping perfusion rates constant. For a given velocity profile, the shear stress is directly proportional to the viscosity of the fluid. Dextran molecules were added to change the viscosity of the flowing fluid while keeping velocity constant, thereby 

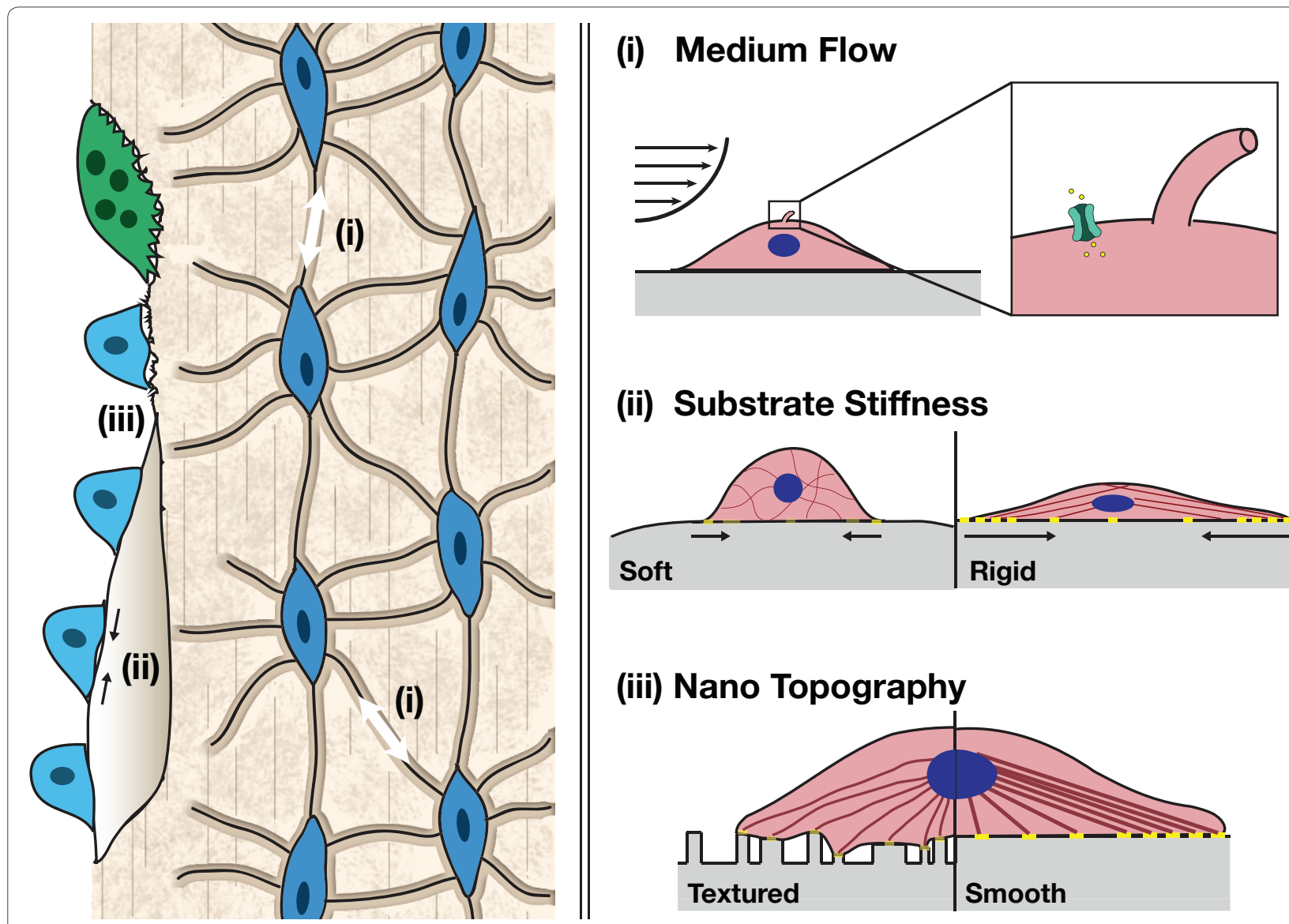

\section{(ii) Substrate Stiffness}

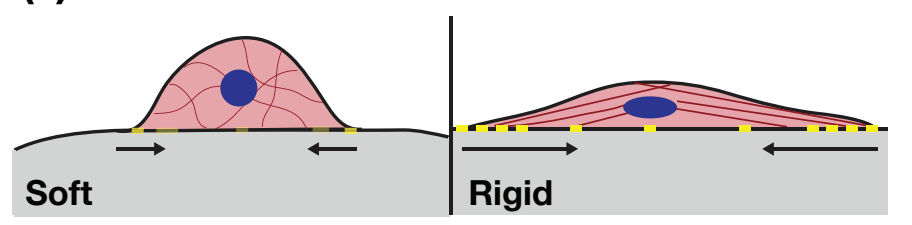

(iii) Nano Topography

Figure 1. Role of mechanical cues on osteogenic differentiation. Left: native bone. Physiological loading of bone induces fluid flow within the canaliculi, resulting in shear stress on the osteocytes (i), which transmit these signals to osteoclasts and osteoblasts to remodel the bone. Stiffness (ii) and topography (iii) of native bone matrix also impacts new bone deposition by osteoblastic cells. Right: tissue engineers apply mechanical stimuli to enhance the osteogenic response of stem and progenitor cells in vitro. (i) Perfusion of culture medium over cell monolayers or through three-dimensional constructs imparts shear stress. Shear induces stretching of the cell membrane, allowing an influx of calcium ions through stretch-activated ion channels. Fluid flow also deflects the primary cilia that extend from the surfaces of osteocytes and osteoblasts, altering signal transduction as a result of microtubule tension. (ii) Substrate rigidity influences cell adhesion, spreading, and differentiation patterns. Soft surfaces provide low resistance, decreased focal adhesion (yellow) strength and reduced cytoskeletal organization relative to more rigid surfaces. This leads to changes in nuclear shape and gene expression. (iii) Topography: surface roughness along with spacing and randomness in nanoscale topographical features influence cell adhesion and the formation of localized stresses along the cell membrane. For example, differences in interfeature $z$-scale dimensions greater than $50 \mathrm{~nm}$ impair local focal adhesion strength. These differences are transmitted to the nucleus via actin filaments and lead to changes in gene expression and cell fate.

increasing shear stress without changing mass transport properties. Grafts exhibited greater matrix and mineral deposition in response to higher viscosity, suggesting that the superior tissue formation characteristics were directly related to increased shear stresses [17]. These threedimensional studies all employed uniform flow rates for the duration of their culture period. Given the dynamic nature of in vivo loads on bone cells, an important area for future studies may be optimizing flow profiles over time (for example, amplitude and frequency of oscillatory and pulsatile patterns) to maximize the formation of new bone.

\section{Scaffold rigidity: role of substrate mechanics}

Cells maintain an interactive, bi-directional signaling relationship with their immediate microenvironment, modifying and organizing the ECM while also directly responding to a plethora of cues provided by the ECM. The biochemical and physical features of the ECM, including the composition and concentration of adhesive ligands, topography, and rigidity impact cellular physiology and influence cell shape, motility, polarization, and cytoskeletal alignment through the formation of focal adhesion complexes. Upon binding the ECM, cells exert contractile forces. The resistance to these forces provides 
information to the cells regarding the compliance of the underlying substrate. Pioneering studies have demonstrated that the rigidity of polyacrylamide gel substrates critically influences the differentiation of MSCs [22]. In particular, MSCs cultured on substrates with elastic moduli mimicking those of brain, muscle, or nonmineralized bone tissues responded by adopting the phenotypic characteristics of neuronal, myogenic, or osteogenic lineages, respectively. The expression of bone markers was highest when MSCs were cultured on the stiffest gels having elastic moduli of approximately $100 \mathrm{kPa}$, which is similar to that measured for nonmineralized bone. It should be noted, however, that while induction media containing traditional biochemical factors directed cells towards a specific lineage only when cultured on substrates within the optimal ranges of stiffness for that tissue, it appeared that substrate stiffness was not itself a sufficiently potent cue to guide undifferentiated stem cells down a given lineage.

Similar results have been reported regarding the osteogenic and adipogenic potential of MSCs: using a system of micrometer-scaled pillars, it was possible to independently regulate cell adhesion (focal adhesion density) and substrate stiffness by controlling the spacing and height, respectively, of the pillars [23]. MSCs cultured in this system were exposed to cocktails of adipogenic and osteogenic factors. It was found that softer surfaces induced a greater adipogenic response, while stiffer surfaces stimulated osteogenic differentiation of cells. The results of both studies conclusively demonstrate that physical characteristics of the matrix environment are critical for the adoption and maintenance of cellular phenotype.

The results from these monolayer studies indicate that it is important to consider the mechanical properties of biomaterial scaffolds used for stem cell-based BTE. Recent studies with silk scaffolds by independent groups have demonstrated that scaffold stiffness influences the composition and mechanics of the resulting tissue grafts. Silk fibroin has been extensively used for tissue engineering applications [24]. It is a naturally derived, biodegradable material and has tunable mechanical properties [25]. In a study using adipose-derived stem cells seeded into scaffolds of different stiffnesses, mechanical tests after 7 weeks of osteogenic culture indicated that the scaffolds with the highest initial stiffness also induced the highest increase in mechanical properties. Similar results were reported in a study using silk scaffolds reinforced with silk microparticles [26]. In this case, MSCs seeded into the stiffest scaffolds exhibited the highest calcium content and expression of bonespecific proteins, but not the greatest increase in bone volume fraction as determined using micro-computed tomography.
While these results demonstrate the impact of scaffold mechanics on the cellular responses, the ultimate mechanical properties of the resulting tissue grafts remain suboptimal for bone applications. For example, the maximum modulus achieved by silk scaffolds after in vitro culture was around $150 \mathrm{kPa}$ while the modulus of native bone may be several orders of magnitude higher. Hence, in vivo applications typically use stiffer scaffolds made of $\beta$-tricalcium phosphate ( $\beta$-TCP) $[27,28]$, hydroxyapatite (HA) $[29,30]$, or even combinations of the two [31]. Incorporating HA into the wall structure of silk scaffolds significantly enhanced the bone tissue formation properties of MSCs cultured in vitro [32]. In this case, HA impacted both the stiffness and biochemical composition of the scaffold. The resulting mineralization structure, however, strongly suggested that the increased wall roughness played an instrumental role in guiding mineral deposition with the HA 'nodes' on the surfaces effectively acting as 'nucleation sites'. Additional studies have demonstrated that topography may also provide mechanical signals that can be transduced directly by cells and influence a number of key cellular processes, including adhesion, contact guidance, cytoskeletal assembly, and gene expression [33].

\section{Mechanical effects of surface topography}

Recent advances in fabrication techniques enable the formation of nano- and micro-scale structural components to study their effects on cellular outcomes. Nanotopographic cues such as pores, ridges, pits, islands, grooves, fibers, and nodes can elicit cell type-dependent behaviors with features as small as $10 \mathrm{~nm}$. Using colloidal lithography to control the application of cylindrical features (100 nm diameter, $160 \mathrm{~nm}$ height, and spaced $230 \mathrm{~nm}$ apart [34]), it was demonstrated that nanotextured substrates limit cell spreading and cytoskeletal organization by inhibiting the formation of robust and dense focal adhesions, resulting in decreased tension on the cytoskeleton. Forces transmitted to the nucleus via the cytoskeleton induce changes in nuclear deformation leading to altered gene expression $[35,36]$.

Consequently, nanomaterials have exhibited considerable ability to regulate cell differentiation and tissue formation characteristics [37]. One landmark study reported that simply by providing disorder to the nanoscaled pillars, it was possible to enhance the expression of osteopontin and osteocalcin in MSCs even in the absence of osteogenic supplements in the culture medium. Cells were cultured on square, hexagonal, disordered (pillars displaced from their position in a square), and random patterned surfaces. Intriguingly, it was found that highly ordered patterns were inhibitory to osteogenesis while displacing the pillars approximately $50 \mathrm{~nm}$ from their ordered geometry enabled statistically significant increases 
in the expression of osteo-specific genes [34]. Other studies have also investigated the effect of cell shape on MSC osteogenic capabilities. A recent study utilized micro-patterned substrates to regulate MSC adhesion and spreading [38]. As a result, BMP-induced osteogenesis was inhibited. This suggests that cellular responses to nanotography might either be directly due to mechanotransduced signals or may be indirectly related to alterations in biological responses due to changes in cell shape.

These reports indicate an additional mechanism for controlling stem cell differentiation and tissue formation properties. They can provide alternatives to invasive inhibition studies to investigate fundamental biological questions. The knowledge gleaned from these studies may then be applied to enhance biomaterials used for regeneration. For example, fibrous capsules often surround bone prostheses and prevent their direct integration with bone tissues. High throughput assays may enable deeper understanding of cell-material interactions and provide insight into how materials might be altered to optimize integration with the host tissues [39].

\section{Mechanical regulation of bone growth in vivo}

Upon transplantation into a host, a milieu of cellular and biochemical factors impact the viability of engineered bone grafts. This complex microenvironment, which includes inflammatory and neo-vascularization responses, significantly affects stem cell differentiation and shapes tissue formation patterns. Additionally, bone grafts implanted into load-bearing sites are subjected to physiological loading. Regulating the temporal (immediate versus delayed) application of these loads affects graft-host integration and impacts tissue formation profiles. A widely studied model of mechanics in bone graft regeneration is the femoral defect in rats. In a study investigating the treatment of $8 \mathrm{~mm}$ defects in rat femurs, a modified alginate scaffold was implanted and engineered to provide controlled release of bone morphogenetic protein 2 (BMP-2). The approach relied on recruitment of the host's osteoprogenitor cells in response to the released growth factor. Internal fixation plates were used to maintain the alignment of the femur. Based on their design and compliance, the plates (i) shielded the grafts from mechanical loads for the entire 12-week implant period, (ii) transferred load to the graft immediately after implantation, or (iii) only after the first 4 weeks of implantation. It was shown that immediately exposing the grafts to sustained physiological loads resulted in scaffold failure by 12 weeks postimplantation. Alternatively, shielding the graft for 4 weeks before exposing them to physiological loads for the subsequent 8 weeks improved bone volume and integration with host tissue relative to the control group (shielded for the duration of the study). These results show the complexity of tissue outcomes in response to temporal mechanical control [40]. In a subsequent study, the identical defect model was used to demonstrate the effect of mechanics on the interplay between bone formation and angiogenesis [41] into the scaffold and provide mechanistic insight into earlier results. Allowing the scaffold to withstand physiological loading immediately upon implantation inhibited vascular ingrowth and subsequent osteogenesis. Alternatively, shielding the scaffolds from loading for the first 4 weeks postimplantation allowed the infiltration of neo-vasculature. Increasing the compliance of the plate at this time allowed invading osteoprogenitor cells to respond to mechanical stresses, leading to an overall enhanced endochondral ossification response compared to control groups [42]. This result corroborates earlier studies where structures resembling secondary ossification centers appeared in the explanted femoral condyles of 5-day old rabbits after exposure to cyclic mechanical loading at $1 \mathrm{~Hz}$ for 12 hours [43]. The potential for using mechanical cues to inhibit bone formation has also been studied using a $1.5 \mathrm{~mm}$ transverse defect in the rat femur [44]. Application of cyclic bending beginning at 10 days postoperation resulted in slowed bone healing and increased cartilage volume, evidenced by histological staining for Safranin $\mathrm{O}$ and gene expression data for cartilage markers collagen II and collagen X. This is consistent with studies demonstrating that the increased cartilage production is actually a prolonged cartilage phase in an endochondral ossification process $[45,46]$.

\section{Conclusion}

Bone tissue engineering makes considerable use of insights from mechanobiology studies and many advances have been made in utilizing mechanics to improve the functionality of bone grafts. Understanding the anatomical structure of native bone and how forces are transmitted to cells has revealed the need to implement fluid-induced shear stress, substrate compliance and topography as biophysical stimuli integral to bone tissue engineering. In three-dimensional in vitro systems, scaffolds typically shield cells from the direct effects of compressive forces, so compression is rarely used to enhance osteogenic outcomes in vitro. However, during fracture healing in vivo, compression may work synergistically (and in a time-dependent manner) with other microenvironmental stimuli, to enhance bone formation via an endochondral ossification pathway.

Scaffold stiffness has profound effects on the osteogenic differentiation of MSCs and in vitro studies have revealed that cells respond to more rigid scaffolds by increasing mineral deposition. Continuing, conventional wisdom suggests that it is desirable to replace 'like with like'; 
hence, bone grafts should have mechanical properties approximating that of native bone to provide immediate functionality upon implantation. The validity of this assumption, however, remains debated as, reportedly, rigid scaffolds do not integrate as readily with host tissues as softer grafts. In examining the role of mechanics on in vivo bone repair, it is not universally accepted that exogenous cells are required and the roles of these 'endogenous' approaches to bone repair have received increasing attention [47].

Future BTE studies will continue to incorporate mechanical considerations to enhance osteogenic differentiation and mineral deposition within grafts. More fundamental understanding of mechanotransduction is nevertheless required to overcome empirical approaches. Non-invasive image-based modalities used to study in vivo bone formation processes in response to specific mechanical stimuli [48] would help to integrate mechanics with other important parameters capable of influencing bone development.

This article is part of a thematic series on Physical influences on stem cells edited by Gordana Vunjak-Novakovic. Other articles in the series can be found online at http://stemcellres.com/series/physical

\section{Abbreviations}

BMP-2 bone morphogenetic protein 2; B-TCP-B-tricalcium phosphate; BTE, bone tissue engineering; COX-2, cyclooxygenase-2; ECM, extracellular matrix; $H A$, hydroxyapatite; OPG, osteoprogerin; RANKL, receptor activator of nuclear factor kB ligand.

\section{Competing interests}

The authors declare that they have no competing interests.

\section{Acknowledgments}

This work was funded by Johns Hopkins University Biomedical Engineering Department and grants from the Maryland Stem Cell Research Fund and the Department of Defense.

\section{Authors' contributions}

$\mathrm{BPH}, \mathrm{DLH}$ and WLG wrote the paper, conceptualized and drew the schematic. All authors read and approved the final manuscript.

\section{Author details}

'Department of Biomedical Engineering, The Johns Hopkins University, Baltimore, MD 21287, USA. ${ }^{2}$ Translational Tissue Engineering Center, The Johns Hopkins University, Baltimore, MD 21231, USA.

Published: 31 January 2013

\section{References}

1. Grayson WL, Martens TP, Eng GM, Radisic M, Vunjak-Novakovic G: Biomimetic approach to tissue engineering. Semin Cell Dev Biol 2009, 20:665-673.

2. Duncan RL, Turner $\mathrm{CH}$ : Mechanotransduction and the functional-response of bone to mechanical strain. Calcif Tissue Int 1995, 57:344-358.

3. Shaw SR, Vailas AC, Grindeland RE, Zernicke RF: Effects of a 1-week spaceflight on morphological and mechanical-properties of growing bone. Am J Physiol 1988, 254:R78-R83.

4. Bassey EJ, Ramsdale SJ: Increase in femoral bone-density in young-women following high-impact exercise. Osteoporos Int 1994, 4:72-75.

5. Rubin J, Fan X, Biskobing DM, Taylor WR, Rubin CT: Osteoclastogenesis is repressed by mechanical strain in an in vitro model. J Orthop Res 1999, 17:639-645.
6. Dehority W, Halloran BP, Bikle DD, Curren T, Kostenuik PJ, Wronski TJ, Shen Y Rabkin B, Bouraoui A, Morey-Holton E: Bone and hormonal changes induced by skeletal unloading in the mature male rat. Am J Physiol 1999, 276:E62-69.

7. Cowin SC, Weinbaum S: Strain amplification in the bone mechanosensory system. Am J Med Sci 1998, 316:184-188.

8. Han Y, Cowin SC, Schaffler MB, Weinbaum S: Mechanotransduction and strain amplification in osteocyte cell processes. Proc Natl Acad Sci U S A 2004, 101:16689-16694.

9. Zeng Y, Cowin SC, Weinbaum S: A fiber matrix model for fluid flow and streaming potentials in the canaliculi of an osteon. Ann Biomed Eng 1994, 22:280-292.

10. Rawlinson SC, Pitsillides AA, Lanyon LE: Involvement of different ion channels in osteoblasts' and osteocytes' early responses to mechanical strain. Bone 1996, 19:609-614.

11. Malone AM, Anderson CT, Tummala P, Kwon RY, Johnston TR, Stearns T, Jacobs CR: Primary cilia mediate mechanosensing in bone cells by a calcium-independent mechanism. Proc Natl Acad Sci U S A 2007, 104:13325-13330.

12. Srinivasan S, Weimer DA, Agans SC, Bain SD, Gross TS: Low-magnitude mechanical loading becomes osteogenic when rest is inserted between each load cycle. J Bone Miner Res 2002, 17:1613-1620.

13. Goodship AE, Lawes TJ, Rubin CT: Low-magnitude high-frequency mechanical signals accelerate and augment endochondral bone repair: preliminary evidence of efficacy. J Orthop Res 2009, 27:922-930.

14. Jacobs CR, Yellowley CE, Davis BR, Zhou Z, Cimbala JM, Donahue HJ: Differential effect of steady versus oscillating flow on bone cells. J Biomech 1998, 31:969-976.

15. Li J, Rose E, Frances D, Sun Y, You L: Effect of oscillating fluid flow stimulation on osteocyte mRNA expression. J Biomech 2012, 45:247-251.

16. Bancroft GN, Sikavitsas VI, van den Dolder J, Sheffield TL, Ambrose CG, Jansen $J A$, Mikos AG: Fluid flow increases mineralized matrix deposition in threedimensional perfusion culture of marrow stromal osteoblasts in a dosedependent manner. Proc Natl Acad SciU S A 2002, 99:12600-12605.

17. Sikavitsas VI, Bancroft GN, Holtorf HL, Jansen JA, Mikos AG: Mineralized matrix deposition by marrow stromal osteoblasts in three-dimensional perfusion culture increases with increasing fluid shear forces. Proc Natl Acad SciU S A 2003, 100:14683-14688.

18. Grayson WL, Marolt D, Bhumiratana S, Frohlich M, Guo XE, Vunjak-Novakovic $\mathrm{G}$ : Optimizing the medium perfusion rate in bone tissue engineering bioreactors. Biotechnol Bioeng 2010, 108:1159-1170.

19. Cartmell SH, Porter BD, Garcia AJ, Guldberg RE: Effects of medium perfusion rate on cell-seeded three-dimensional bone constructs in vitro. Tissue Eng 2003, 9:1197-1203.

20. Porter BD, Lin AS, Peister A, Hutmacher D, Guldberg RE: Noninvasive image analysis of three-dimensional construct mineralization in a perfusion bioreactor. Biomaterials 2007, 28:2525-2533.

21. Grayson WL, Frohlich M, Yeager K, Bhumiratana S, Chan ME, Cannizzaro C, Wan LQ, Liu XS, Guo XE, Vunjak-Novakovic G: Engineering anatomically shaped human bone grafts. Proc Natl Acad Sci U S A 2010, 107:3299-3304.

22. Engler AJ, Sen S, Sweeney HL, Discher DE: Matrix elasticity directs stem cell lineage specification. Cell 2006, 126:677-689.

23. Yim EK, Reano RM, Pang SW, Yee AF, Chen CS, Leong KW: Nanopatterninduced changes in morphology and motility of smooth muscle cells. Biomaterials 2005, 26:5405-5413.

24. Garcia-Fuentes M, Meinel AJ, Hilbe M, Meinel L, Merkle HP: Silk fibroin/ hyaluronan scaffolds for human mesenchymal stem cell culture in tissue engineering. Biomaterials 2009, 30:5068-5076.

25. Kim HJ, Kim HS, Matsumoto A, Chin IJ, Jin HJ, Kaplan DL: Processing windows for forming silk fibroin biomaterials into a three-dimensional porous matrix. Aust J Chem 2005, 58:716-720.

26. Rockwood DN, Gil ES, Park SH, Kluge JA, Grayson W, Bhumiratana S, Rajkhowa R, Wang XG, Kim SJ, Vunjak-Novakovic G, Kaplan DL: Ingrowth of human mesenchymal stem cells into porous silk particle reinforced silk composite scaffolds: An in vitro study. Acta Biomaterialia 2011, 7:144-151.

27. Umeda H, Kanemaru S, Yamashita M, Kishimoto M, Tamura Y, Nakamura T, Omori K, Hirano S, Ito J: Bone regeneration of canine skull using bone marrow-derived stromal cells and beta-tricalcium phosphate. Laryngoscope 2007, 117:997-1003.

28. Nienhuijs MEL, Walboomers XF, Briest A, Merkx MAW, Stoelinga PJW, Jansen JA: Healing of bone defects in the goat mandible, using COLLOSS (R) E and 
beta-tricalciumphosphate. J Biomed Mater Res 2010, 92B:517-524.

29. Azami M, Samadikuchaksaraei A, Poursamar SA: Synthesis and characterization of a laminated hydroxyapatite/gelatin nanocomposite scaffold with controlled pore structure for bone tissue engineering. Int J Artif Organs 2010, 33:86-95.

30. Nitzsche H, Lochmann A, Metz H, Hauser A, Syrowatka F, Hempel E, Muller T, Thurn-Albrecht T, Mader K: Fabrication and characterization of a biomimetic composite scaffold for bone defect repair. $J$ Biomed Mater Res 2010, 94A:298-307.

31. Ramay HR, Zhang M: Biphasic calcium phosphate nanocomposite porous scaffolds for load-bearing bone tissue engineering. Biomaterials 2004 25:5171-5180

32. Bhumiratana S, Grayson WL, Castaneda A, Rockwood DN, Gil ES, Kaplan DL, Vunjak-Novakovic G: Nucleation and growth of mineralized bone matrix on silk-hydroxyapatite composite scaffolds. Biomaterials 2011, 32:2812-2820.

33. Prager-Khoutorsky M, Lichtenstein A, Krishnan R, Rajendran K, Mayo A, Kam Z, Geiger B, Bershadsky AD: Fibroblast polarization is a matrix-rigiditydependent process controlled by focal adhesion mechanosensing. Nat Cell Biol 2011, 13:1457-U1178.

34. Dalby MJ, Gadegaard N, Tare R, Andar A, Riehle MO, Herzyk P, Wilkinson CD, Oreffo RO: The control of human mesenchymal cell differentiation using nanoscale symmetry and disorder. Nat Materials 2007, 6:997-1003.

35. McGrail DJ, Ghosh D, Quach ND, Dawson MR: Differential mechanical response of mesenchymal stem cells and fibroblasts to tumor-secreted soluble factors. PLoS One 2012, 7:e33248.

36. Knight MM, van de Breevaart Bravenboer J, Lee DA, van Osch GJ, Weinans H, Bader DL: Cell and nucleus deformation in compressed chondrocytealginate constructs: temporal changes and calculation of cell modulus. Biochimica et Biophysica Acta 2002, 1570:1-8.

37. McNamara LE, MCMurray RJ, Biggs MJ, Kantawong F, Oreffo RO, Dalby MJ: Nanotopographical control of stem cell differentiation. J Tissue Eng 2011, 2010:120623

38. Wang YK, Yu X, Cohen DM, Wozniak MA, Yang MT, Gao L, Eyckmans J, Chen CS: Bone morphogenetic protein-2-induced signaling and osteogenesis is regulated by cell shape, RhoA/ROCK, and cytoskeletal tension. Stem Cells Dev 2012, 21:1176-1186.
39. Unadkat HV, Hulsman M, Cornelissen K, Papenburg BJ, Truckenmuller RK, Carpenter AE, Wessling M, Post GF, Uetz M, Reinders MJ, Stamatialis D, van Blitterswijk CA, de Boer J: An algorithm-based topographical biomaterials library to instruct cell fate. Proc Natl Acad Sci U S A 2011, 108:16565-16570.

40. Boerckel JD, Dupont KM, Kolambkar YM, Lin AS, Guldberg RE: In vivo model for evaluating the effects of mechanical stimulation on tissue-engineered bone repair. J Biomech Eng 2009, 131:084502.

41. Szpalski C, Barbaro M, Sagebin F, Warren SM: Bone tissue engineering: current strategies and techniques - part II: cell types. Tissue Eng Part B Rev 2012, 18:258-269.

42. Boerckel JD, Uhrig BA, Willett NJ, Huebsch N, Guldberg RE: Mechanical regulation of vascular growth and tissue regeneration in vivo. Proc Natl Acad Sci U S A 2011, 108:E674-680.

43. Sundaramurthy S, Mao JJ: Modulation of endochondral development of the distal femoral condyle by mechanical loading. J Orthop Res 2006, 24:229-241.

44. Palomares KT, Gleason RE, Mason ZD, Cullinane DM, Einhorn TA, Gerstenfeld LC, Morgan EF: Mechanical stimulation alters tissue differentiation and molecular expression during bone healing. J Orthop Res 2009 27:1123-1132.

45. Epari DR, Schell H, Bail HJ, Duda GN: Instability prolongs the chondral phase during bone healing in sheep. Bone 2006, 38:864-870.

46. Boerckel JD, Kolambkar YM, Stevens HY, Lin AS, Dupont KM, Guldberg RE: Effects of in vivo mechanical loading on large bone defect regeneration. J Orthop Res 2012, 30:1067-1075.

47. Hutmacher DW, Duda G, Guldberg RE: Endogenous musculoskeletal tissue regeneration. Cell Tissue Res 2012, 347:485-488.

48. Tuan HS, Hutmacher DW: Application of micro CT and computation modeling in bone tissue engineering. Computer-Aided Design 2005, 37:1151-1161

\section{doi:10.1186/scrt158}

Cite this article as: Hung BP, et al: Mechanical control of tissue-engineered bone. Stem Cell Research \& Therapy 2013, 4:10. 